\title{
Matched-Filter based Backscatter Communication for IoT Devices over Ambient OFDM Carrier
}

\author{
Jinho Choi
}

\begin{abstract}
In this paper, we study backscatter communication (BC) for power-limited devices that are connected to a network for the Internet of Things (IoT), where joint estimation and detection is carried out at a receiver to detect signals from a backscatter device (BD) with ambient orthogonal frequency division multiplexing (OFDM) carrier. In conventional $\mathrm{BC}$, in order to avoid the difficulty of the carrier estimation, the energy detector is usually considered at a receiver at the cost of poor performance. To improve the performance, in this paper, we consider a novel approach that allows the carrier estimation at the receiver via joint estimation and detection. In particular, in the proposed approach, the matched-filtering at the BD (for the transmitter filter) is employed to impose a certain property that allows efficient and reliable carrier estimation via joint estimation and detection. Through the performance analysis and simulation results, we show that the matched-filtering at the $B D$ in the proposed approach can improve the performance.
\end{abstract}

Index Terms-Internet of Things; backscatter communication; OFDM; carrier estimation

\section{INTRODUCTION}

The Internet of Things (IoT) has attracted attention from both academia and practitioners and is considered for standardizations [1] [2]. The IoT is to connect any device to the Internet through wired or wireless communications for various applications and services. While some devices might be quite capable, e.g., smart phones, most cheap IoT devices would have various limitations. In particular, some IoT devices may rely on the power from radio frequency (RF) signals, i.e., RF powered devices, to compute and communicate with other devices [3]. In this case, backscatter communication would play a crucial role in transmitting information to nearby receivers such as RF identification (RFID) systems [4] [5]. There are a number applications of RFID technology. For example, in [6] and [7], RFID is used for inventory and localization, respectively. In addition, in [8], for networking with miniature medical sensors or devices implanted in deep tissues, RFID technology is considered.

While RFID requires dedicated RF transmitters, it is also possible to exploit other existing or ambient RF signals [9]. Ambient backscatter communication (AmBC) has been studied for devices of limited power (such as sensors), which are called backscatter devices (BDs), in the IoT to transmit their information to nearby receivers by exploiting various ambient RF signals [9] [10] [11]. For the source of ambient RF signals, TV and WiFi signals can be considered [9] [12]. In [13], a ambient backscatter technique using multiple antennas is studied to achieve a high data rate in AmBC. In [14], a

The author is with School of Information Technology, Deakin University, Geelong, VIC 3220, Australia. (e-mail: jinho.choi@deakin.edu.au). cooperative $\mathrm{AmBC}$ approach is investigated where a reader recovers information not only from a $\mathrm{BD}$, but also from an RF source. In [15], a full-duplex based AmBC network is proposed where an access point (AP) can simultaneously transmits downlink OFDM signals to its user and receives uplink signals backscattered from multiple BDs.

In $\mathrm{AmBC}$, there are various challenges such as direct-link interference. Unlike RFID systems, an ambient signal is not a tone, but a modulated signal. Provided that the ambient signal is a narrowband signal, in [11], differential modulation scheme is employed for backscattered signals so that the energy of direct-link interference can be mitigated. If the ambient signal is not a narrowband signal (e.g., WiFi signals) and experiences frequency-selective fading, the mitigation of wideband directlink interference becomes more challenging. In [12], the notion of BackFi is introduced where a WiFi AP is not only a reader (or receiver for backscattered signals), but also an ambient transmitter. Since the AP transmits signals (which are ambient signals for a BD), it can employ self-interference-cancellation using reflected signals. However, if the ambient transmitter and the receiver are different, the mitigation of wideband direct-link interference becomes hard as the receiver cannot directly observe the channel state information (CSI) from the ambient transmitter to a BD. To avoid this problem, in [16], a special structure of orthogonal frequency division multiplexing (OFDM) signals, which is called the repeating structure, is exploited to suppress wideband direct-link interference in detecting backscattered signals.

Ambient OFDM carrier [16] is an attractive approach for backscatter communication as OFDM systems are popular, e.g., long-term evolution (LTE) systems for cellular communications [17], WiFi, and digital video broadcasting - terrestrial (DVB-T) [18]. Thus, in this paper, we consider ambient OFDM carrier. However, unlike [16], we do not rely on the repeating structure of OFDM signals. The reasons are twofold: $i$ ) The repeating structure is due to cyclic prefix (CP). If the length of $\mathrm{CP}$ is not sufficiently long, it is not possible to exploit the repeating structure. $i i)$ When the repeating structure is used to detect backscattered signals, the performance is independent of the length of OFDM symbols. However, in general, a better performance is expected if the length of OFDM symbols increases as the bit energy of backscattered signals increases.

In this paper, we consider coherent detection of backscattered signals via joint estimation and detection for a better detection performance in AmBC. Note that although coherent detection can provide a better performance than noncoherent detection (which is widely adopted to detect backscattered signals) [19], it has more challenges including the carrier 
estimation. For the carrier estimation, the receiver needs to know BD's CSI, which is not directly observable at the receiver. As a result, in conventional approaches, reliable carrier estimation becomes difficult as the receiver cannot directly estimate BD's CSI. To address this challenge, we propose the matched-filtering at the $\mathrm{BD}$ (for the transmitter filter) to impose a certain property that allows efficient and reliable carrier estimation via joint estimation and detection at the receiver. Furthermore, since joint estimation and detection at the receiver usually requires a high-computational complexity, we consider an iterative method based on the expectationmaximization (EM) algorithm [20] [21], where the complexity of each iteration is linearly proportional to the length of OFDM symbol. The number of iterations to convergence is also a few (at a high signal-to-noise ratio (SNR), 5 iterations are shown to be sufficient).

The work in this paper differs from other existing works. Unlike [12], it does not require that the ambient transmitter is also the receiver to mitigate wideband direct-link interference. Furthermore, as opposed to [16], the proposed approach is applicable even if the length of $\mathrm{CP}$ is short. since the repeating structure is not employed. In [22], as in this paper, the estimation of CSI is considered for semi-coherent detection. However, only flat fading channels are considered in [22], while frequency-selective fading channels are assumed in this paper. In addition, we consider full coherent detection.

The rest of the paper is organized as follows. In Section II. we present an OFDM-based AmBC model and discuss difficulties due to wideband direct-link interference. Existing approaches to detect backscattered signals with ambient OFDM carrier are presented in Section III. We propose a new approach that effectively allows coherent detection of scattered signals by taking advantage of known CSI and present a low-complexity joint estimation and detection method in Section IV A performance analysis is presented in Section V. In Section VI we present simulation results. The paper is concluded with some remarks in Section VII

Notation: Matrices and vectors are denoted by upper- and lower-case boldface letters, respectively. The superscripts $\mathrm{T}$ and $\mathrm{H}$ denote the transpose and complex conjugate, respectively. The 2-norm of a vector $\mathbf{a}$ is denoted by $\|\mathbf{a}\|$. The Frobenius norm of a matrix $\mathbf{A}$ is denoted by $\|\mathbf{A}\|_{\mathrm{F}} . \mathbb{E}[\cdot]$ and $\operatorname{Var}(\cdot)$ denote the statistical expectation and variance, respectively. $\mathcal{C N}(\mathbf{a}, \mathbf{R})$ represents the distribution of circularly symmetric complex Gaussian (CSCG) random vectors with mean vector a and covariance matrix $\mathbf{R}$. The Q-function is given by $\mathcal{Q}(x)=\int_{x}^{\infty} \frac{1}{\sqrt{2 \pi}} e^{-\frac{t^{2}}{2}} d t$.

\section{SySTEM MODEL}

Consider a system consisting of a legacy (or primary) OFDM transmitter as an RF source (i.e., an ambient transmitter), a $\mathrm{BD}$, and a receiver that is a reader for the $\mathrm{BD}$ as in [16]. The system is illustrated as in Fig. 11. As discussed in [12], there are IoT gadgets such as cheap wearable devices and sensors that can measure physical variables to upload to the cloud through a gateway. These gadgets and devices are to operate for a long time without requiring battery replacements or without batteries. To this end, exploiting ambient signals such as WiFi signals from nearby APs to transmit their information becomes crucial. According to the above scenario, in Fig. 1, the primary OFDM transmitter, the BD, and the receiver are a WiFi AP, a cheap IoT gadget, and a gateway, respectively. Note that as shown in Fig. 1, the receiver is not able to observe or estimate BD's CSI, which is denoted by G, while it can estimate its CSI, which is denoted by $\mathbf{H}$. We will explain $\mathbf{H}$ and $\mathbf{G}$ later.

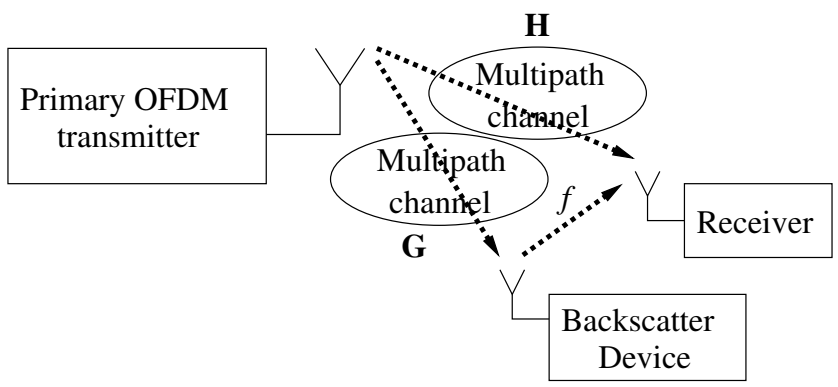

Fig. 1. An illustration of the system consisting of the legacy OFDM transmitter, BD, and receiver. Here, $\mathbf{H}$ and $\mathbf{G}$ represent the frequency-domain (wideband) channel matrices from the legacy OFDM transmitter to the receiver and the $\mathrm{BD}$, respectively, while $f$ represents the channel coefficient from the $\mathrm{BD}$ to the receiver.

For convenience, the system consisting of a legacy OFDM transmitter and legacy receivers is referred to as the legacy system. Denote by $\left\{h_{p}\right\}$ the channel impulse response (CIR) from the legacy OFDM transmitter to the receiver and by $\left\{g_{p}\right\}$ the CIR the legacy OFDM transmitter to the BD. Since the bandwidth of the legacy OFDM system is wide, the lengths of CIRs are relatively long and the channels experience intersymbol-interference (ISI) and become frequency-selective [19], [23]. Let $\mathbf{x}_{m}$ denote the $m$ th (ambient) OFDM symbol transmitted by the legacy OFDM transmitter. We assume that the length of $\mathbf{x}_{m}$ is $L$, i.e., $\mathbf{x}_{m}=\left[\begin{array}{lll}x_{0, m} & \ldots & x_{L-1, m}\end{array}\right]^{\mathrm{T}}$, where $x_{l, m}$ represents the signal transmitted through the $l$ th subcarrier. Throughout the paper, we assume that

$$
\mathbb{E}\left[x_{l, m}\right]=0, \operatorname{Var}\left(x_{l, m}\right)=E_{x}, \text { and } x_{l, m} \in \mathcal{X},
$$

where $\mathcal{X}$ is the signal constellation of $x_{l, m}$. In order to deal with the ISI, CP is added [23] [24]. At the receiver, the received signal after removing $\mathrm{CP}$ (in the frequency-domain) becomes

$$
\mathbf{y}_{m}=\mathbf{H} \mathbf{x}_{m}+\mathbf{a}_{m}+\mathbf{n}_{m}, m=0, \ldots, M-1,
$$

where $\mathbf{H}=\operatorname{diag}\left(H_{0}, \ldots, H_{L-1}\right), \mathbf{a}_{m}$ denotes the received signal from the BD (i.e., the backscattered signal), $\mathbf{n}_{m} \sim$ $\mathcal{C N}\left(0, N_{0} \mathbf{I}\right)$ is the background noise vector, and $M$ represents the number of OFDM symbols in a slot. Here, we assume that the length of slot is shorter than the coherence time so that the channel variation over the time is negligible and $H_{l}$ is the channel coefficient of the $l$ th subcarrier, which is given by

$$
H_{l}=\sum_{p=0}^{P_{h}-1} h_{p} e^{-\frac{j 2 \pi l p}{L}}, l=0, \ldots, L-1,
$$

where $P_{h}$ represents the length of the CIR from the legacy OFDM transmitter to the receiver. Similarly, the channel 
coefficient of the $l$ th subcarrier of the channel from the legacy OFDM transmitter to the $\mathrm{BD}$ is given by

$$
G_{l}=\sum_{p=0}^{P_{g}-1} g_{p} e^{-\frac{j 2 \pi l p}{L}}, l=0, \ldots, L-1,
$$

where $P_{g}$ represents the length of the CIR from the legacy OFDM transmitter to the BD.

We can assume that the receiver is sufficiently close to the $\mathrm{BD}$ and the channel from the $\mathrm{BD}$ to the receiver can be modeled as a flat fading channel [16] [12], while the channels from the legacy OFDM transmitters to the $\mathrm{BD}$ and receiver are modeled as frequency-selective fading channels as shown above (due to multiple paths). Consequently, although the system bandwidth is wide, the channel coefficients for all the subcarriers from the BD to the receiver are the same and denoted by $f_{l}=f, l=0, \ldots, L-1$. We can have

$$
\mathbf{a}_{m}=\beta \mathbf{G} \mathbf{X}_{m} \mathbf{b}_{m},
$$

where $\beta=\alpha f, \mathbf{G}=\operatorname{diag}\left(G_{0}, \ldots, G_{L-1}\right), \mathbf{X}_{m}=\operatorname{diag}\left(\mathbf{x}_{m}\right)$, and $\mathbf{b}_{m}$ is the Fourier transform of the BD's baseband signal to be transmitted to the receiver. Here, $\alpha$ is the reflection coefficient that is usually small [4]. In (2), the diagonal elements of $\mathbf{G} \mathbf{X}_{m}$ (i.e., $\mathbf{G} \mathbf{x}_{m}$ ) is the received signal in the frequency-domain at the $\mathrm{BD}$, which is regarded as the Fourier transform of the baseband representation of the carrier that is to be exploited by the BD for backscatter communication. For convenience, denote by $\tilde{\mathbf{b}}_{m}$ and $\tilde{\mathbf{c}}_{m}$ the time-domain representations of $\mathbf{b}_{m}$ and $\mathbf{G} \mathbf{x}_{m}$, respectively. Then, the timedomain representation of $\mathbf{a}_{m}$ is given by

$$
\tilde{\mathbf{a}}_{m}=\beta \tilde{\mathbf{c}}_{m} \otimes \tilde{\mathbf{b}}_{m},
$$

where $\otimes$ represents the convolution. The modulation in (2) is called "modulation in the air" in [16], where $\mathbf{G} \mathbf{x}_{m}$ or $\mathbf{G X} \mathbf{X}_{m}$ becomes the carrier for backscatter communication. Then, the received signal, $\mathbf{y}_{m}$, in (1) is rewritten as

$$
\begin{aligned}
\mathbf{y}_{m} & =\mathbf{H} \mathbf{x}_{m}+\mathbf{a}_{m}+\mathbf{n}_{m} \\
& =\mathbf{H} \mathbf{x}_{m}+\beta \mathbf{G} \mathbf{X}_{m} \mathbf{b}_{m}+\mathbf{n}_{m}, m=0, \ldots, M-1 .
\end{aligned}
$$

We have some remarks as follows.

- With ambient OFDM carrier, as shown in (3), there are two main difficulties to detect backscattered signals. The first difficulty, denoted by Difficulty-I, is due to unknown carrier, $\mathbf{H} \mathbf{x}_{m}$. For the carrier estimation to address the first difficulty, the receiver can exploit pilot signals from the legacy transmitter, which are transmitted prior to transmissions of OFDM (data) symbols so that legacy receivers are able to estimate CSI. Thus, the receiver can easily estimate $\mathbf{H}$. However, it is still required to estimate $\mathbf{x}_{m}$ for the carrier estimation. The second difficulty, denoted by Difficulty-II, is due to unknown BD's CSI, $\mathbf{G}$, which is not directly observable at the receiver.

- The CIRs, $\left\{h_{p}\right\}$ and $\left\{g_{p}\right\}$, include the propagation delays. Thus, the first few coefficients can be zero. In addition, the length of CP should be greater than or equal to the length of CIR, $P_{h}$, to avoid any ISI between adjacent OFDM symbols. However, it is also expected to make the length of $\mathrm{CP}$ as short as possible to maximize the spectral efficiency. Thus, the length of $\mathrm{CP}$ might be the same as the (maximum) length of CIR.

- Since there might be the background or thermal noise at the BD, the transmitted signal from the BD needs to include the background noise. However, the thermal noise is negligible as the $\mathrm{BD}$ might have passive components and limited signal processing operations [11].

\section{EXISTING APPROACHES}

As mentioned earlier, the carrier estimation might be necessary to detect backscattered signals in AmBC. However, there are few existing approaches to avoid the carrier estimation. We briefly explain them in this section together with their shortcomings.

\section{A. With Repeating Structure}

In this subsection, we briefly discuss the approach studied in [16] and explain why this approach cannot be used if there is no additional guard interval between OFDM symbols in a slot.

In [16], a transceiver design is considered by exploiting certain properties of OFDM signals with CP. For simplicity, let $M=1$ and omit the OFDM symbol index $m$ in a slot. Consider the received signal in the time-domain at the receiver without backscattered signals as follows:

$$
\tilde{y}_{t}=\sum_{p=0}^{P_{h}-1} h_{p} \tilde{x}_{t-p}+\tilde{n}_{t}, t=0, \ldots, L+L_{\mathrm{cp}}-1,
$$

where $L_{\mathrm{cp}}$ represents the length of CP and $\tilde{x}_{t}$ and $\tilde{n}_{t}$ denote the $t$ th signal and noise at the time-domain, respectively. As $\mathrm{CP}$ is inserted, we have [24] [23]

$$
\tilde{x}_{t}=\tilde{x}_{L+t}, t \in\left\{0, \ldots, L_{\mathrm{cp}}-1\right\} .
$$

The above repetition results in the following property called the repeating structure in [16]:

$$
\tilde{y}_{t}=\tilde{y}_{L+t}, t \in\left\{P_{h}, \ldots, L_{\mathrm{cp}}-1\right\},
$$

which plays a key role in deriving the transceiver in [16].

Let $\tilde{b}_{t}$ denote the signal transmitted by the BD in the timedomain. To exploit the repeating structure in (4), the following signal is considered in [16]:

$$
\begin{aligned}
& \text { Bit } 0: \tilde{b}_{t}=1, t=\left\{0, \ldots, L+L_{\mathrm{cp}}-1\right\} \\
& \text { Bit } 1: \tilde{b}_{t}=\left\{\begin{aligned}
1, & \text { if } t=\{0, \ldots, \bar{L}\} \\
-1, & \text { if } t=\left\{\bar{L}, \ldots, L+L_{\mathrm{cp}}-1\right\},
\end{aligned}\right.
\end{aligned}
$$

where $\bar{L}=\left\lfloor\frac{L+L_{\mathrm{cp}}-1}{2}\right\rfloor$. With the signal transmitted from the $\mathrm{BD}$, the received signal at the receiver in the time-domain becomes

$$
\tilde{y}_{t}=h_{t} \otimes \tilde{x}_{t}+\beta\left(g_{t} \otimes \tilde{x}_{t}\right) \tilde{b}_{t}+\tilde{n}_{t}
$$

Let

$$
\tilde{d}_{t}=\tilde{y}_{t}-\tilde{y}_{L+t}, t \in\left\{P_{h}, \ldots, L_{\mathrm{cp}}-1\right\} .
$$

Then, if bit 0 is transmitted from the BD, we have

$$
\tilde{d}_{t}=\tilde{e}_{t} \triangleq \tilde{n}_{t}-\tilde{n}_{t+L}, t \in\left\{P_{h}, \ldots, L_{\mathrm{cp}}-1\right\},
$$


where the signals from the legacy OFDM transmitter are canceled thanks to (4). On the other hand, if bit 1 is transmitted, it follows

$$
\tilde{d}_{t}=2\left(g_{t} \otimes \tilde{x}_{t}\right)+\tilde{e}_{t}, t \in\left\{P_{h}, \ldots, L_{\mathrm{cp}}-1\right\},
$$

where the signal from the legacy OFDM transmitter exists. Thus, the energy detector [25] can be used to detect the signal from the BD as in [16].

Unfortunately, since the approach in [16] relies on the repeating structure in (4), there are few difficulties. Clearly, it requires that $L_{\mathrm{cp}}>P_{h}$ and a better performance can be achieved as $L_{\mathrm{cp}}$ increases, which results in an inefficient use of bandwidth (or subcarriers) and undesirable as mentioned earlier. Another difficulty is that (4) is only valid when $M=1$ or there are additional guard intervals between OFDM symbols for the case of $M>1$. If $M>1$, the received signals corresponding $\mathrm{CP}$ include interference from the previous OFDM symbol, which cannot guarantee (4) due to the repeating structure unless the length of $\mathrm{CP}$ is sufficiently longer than the length of CIR. Note that since CP is used to avoid ISI, additional guard intervals might be redundant to the legacy system and would not be allowed. Consequently, in this paper, we do not further consider the repeating structure.

\section{B. Without Repeating Structure}

Without the repeating structure, we may consider the approach in [11], which is proposed for a narrowband system, with a modification for a wideband system (i.e., an OFDM system).

In order to transmit one bit per OFDM symbol (duration) as in [16], we assume that

$$
\mathbf{b}_{m}=\mathbf{1} s_{m},
$$

where 1 represents a vector of all 1's and $s_{m}$ is a binary signal. For example, we have $s_{m} \in \mathcal{S}=\{-1,+1\}$, while $\mathcal{S}=\{0,1\}$ for noncoherent communication in [11]. Then, the received signal (in the frequency-domain) at the receiver becomes

$$
\mathbf{y}_{m}=\left(\mathbf{H}+\beta \mathbf{G} s_{m}\right) \mathbf{x}_{m}+\mathbf{n}_{m} .
$$

To avoid Difficulty-I (i.e., no carrier estimation is considered at the receiver), it has to be assumed that $\mathbf{H x}_{m}$ is unknown. In addition, $\beta \mathbf{G}$ is also unknown. In this case, with $s_{m} \in\{0,1\}$, the energy detector can be employed with test statistic $\left\|\mathbf{y}_{m}\right\|^{2}$ and a decision threshold ${ }^{1}$ as in [11]. In particular, with $\mathcal{S}=\{0,1\}$, we have

$$
\left\|\mathbf{y}_{m}\right\|^{2}= \begin{cases}\left\|\mathbf{H} \mathbf{x}_{m}+\mathbf{n}\right\|^{2}, & \text { if } s_{m}=0 \\ \left\|(\mathbf{H}+\beta \mathbf{G}) \mathbf{x}_{m}+\mathbf{n}\right\|^{2}, & \text { if } s_{m}=1\end{cases}
$$

Then, the hypothesis test in the energy detector can be carried out as follows:

$$
\left\|\mathbf{y}_{m}\right\|^{2} \underset{\mathcal{H}_{0}}{\stackrel{\mathcal{H}_{1}}{>}} \tau
$$

\footnotetext{
${ }^{1}$ Finding an optimal threshold requires a careful performance analysis to minimize error probabilities as shown in [11]. In addition, the optimal threshold depends on statistical properties of channels, which may not be available in advance.
}

where $\tau$ is a decision threshold, and $\mathcal{H}_{0}$ and $\mathcal{H}_{1}$ denote the hypotheses for $s_{m}=0$ and 1 , respectively. Although this approach does not require the carrier estimation and the estimation of $\mathbf{G}$, its performance is usually poor. For a better performance, we can exploit the knowledge of $\mathbf{H}$ (as mentioned earlier, the receiver is able to estimate $\mathbf{H}$ ) to partially overcome Difficulty-I and consider joint estimation and detection. This approach will be explained in Section IV

\section{MATChED-FILTER BASED TRANSMission AND LOW-COMPLEXITY DETECTION}

In this section, in order to overcome the two main difficulties (i.e., Difficulty-I and Difficulty-II), we discuss a new approach for modulation in the air and derive a low-complexity joint estimation and detection method for the receiver.

\section{A. BD's Transmitter Filter with CSI}

Due to the pilot signal transmitted from the legacy OFDM transmitter, the BD is also able to estimate its CSI, G, or the CIR, $\left\{g_{p}\right\}$, in the time-domain. For convenience, the CIR is also denoted by $g(t)$. Thus, $\left\{g_{p}\right\}$ can be seen as the sampled (or discrete-time) version of $g(t)$. Then, the BD can take advantage of known CSI to help the receiver in order to improve the performance of joint estimation and detection. In particular, we propose the matched-filter based transmission enforcing the forwarded OFDM signal by the $\mathrm{BD}$ to be coherently received at the receiver.

Suppose that the time-reversal and complex conjugate CIR is used as the filter coefficients of the transmitter filter at the $\mathrm{BD}$, which becomes the matched-filtering (to its CSI, $\mathbf{G}$ or $g(t)$ ). Consequently, when the BD is to transmit one bit per OFDM symbol, the baseband signal in the frequency-domain is represented by

$$
\mathbf{b}_{m}=\left\{\begin{aligned}
\kappa \mathbf{g}^{*}, & \text { if Bit } 0 \text { is transmitted } \\
-\kappa \mathbf{g}^{*}, & \text { if Bit } 1 \text { is transmitted, }
\end{aligned}\right.
$$

where $\mathbf{g}=\left[\begin{array}{lll}G_{0} & \ldots & G_{L-1}\end{array}\right]^{\mathrm{T}}$ and $\kappa=\frac{\sqrt{L}}{\|\mathbf{g}\|}$ so that $\left\|\mathbf{b}_{m}\right\|^{2}=L$. Then, it can be shown that

$$
\begin{aligned}
\mathbf{a}_{m} & =\beta \kappa \mathbf{G} \mathbf{X}_{m} \mathbf{g}^{*} s_{m} \\
& =\tilde{\beta} \mathbf{V} \mathbf{x}_{m} s_{m}, s_{m} \in\{-1,+1\} .
\end{aligned}
$$

where $\mathbf{V}=\operatorname{diag}\left(\left|G_{0}\right|^{2}, \ldots,\left|G_{L-1}\right|^{2}\right)$ and $\tilde{\beta}=\beta \kappa$. From (1), $\mathbf{y}_{m}$ becomes

$$
\mathbf{y}_{m}=\left(\mathbf{H}+\tilde{\beta} \mathbf{V} s_{m}\right) \mathbf{x}_{m}+\mathbf{n}_{m} .
$$

The resulting approach with $(10)$ is referred to as the channelaware matched-filter (CAMF) scheme. On the other hand, for convenience, the approach that does not exploit the CSI in (8) with $s_{m} \in\{-1,+1\}$ is referred to as the channel-unaware impulse-filter (CUIF) scheme.

In Fig. 2, the backscattered signals in the time-domain, which are denoted by $\tilde{b}_{m}(t)$ that can be obtained by the inverse Fourier transform of $\mathbf{b}_{m}$, are illustrated with two different transmitter filters. For the CAMF scheme, the BD estimates the CIR, $\left\{g_{p}\right\}$ or $g(t)$. Then, the impulse response of the transmitter filter is $g^{*}\left(T_{s}-t\right)$ and $\tilde{b}_{m}(t)=g^{*}\left(T_{s}-t\right) s_{m}$, 
where $T_{s}$ denotes the OFDM symbol duration. On the other hand, in the CUIF scheme, we have $\tilde{b}_{m}(t)=s_{m} \delta(t)$, i.e., the transmitter filter is the impulse filter. Thus, the corresponding signal in the frequency-domain can be represented as in 8. Compared with CUIF, CAMF requires more signal processing operations for the channel estimation and transmitter filter at the $\mathrm{BD}$ due to the matched-filtering. However, since all the elements of $\mathbf{V},\left\{\left|G_{l}\right|^{2}\right\}$, are non-negative real variables, this property can be exploited at the receiver to overcome Difficulty-II. From this, CAMF can outperform CUIF thanks to the matched-filtering. Note that the signal energy (per bit) for backscatter communication, $\left\|\mathbf{b}_{m}\right\|^{2}$, in the CAMF is the same as that of CUIF scheme, which is $L$.

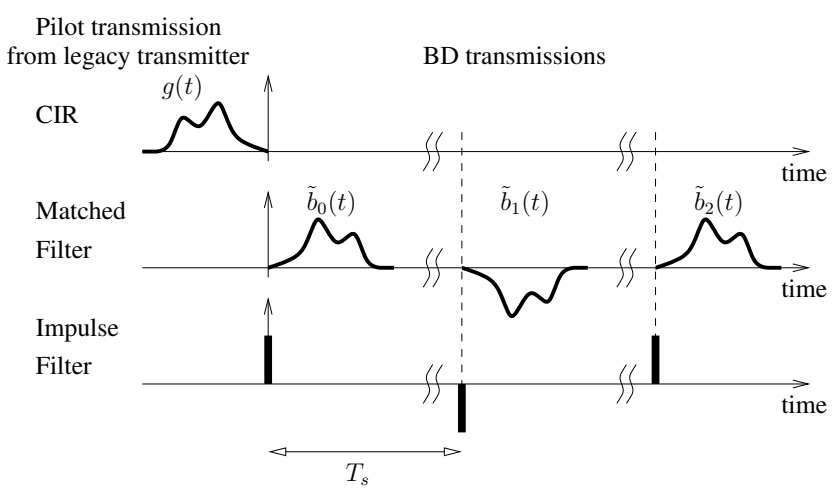

Fig. 2. Illustrations of the backscattered signals in the time-domain with two different transmitter filters.

\section{B. Joint Demodulation and EM Algorithm}

In this subsection, we consider a joint demodulation method to estimate $\mathbf{V}$ (or $\mathbf{G}$ ) as well as to detect backscattered signals using the EM algorithm [20] [21] [24].

Consider the CAMF scheme. For given $\mathbf{y}_{m}$, the likelihood function from (12) can be given by

$$
f\left(\mathbf{y}_{m} \mid \mathbf{x}_{m}, s_{m}, \tilde{\beta}, \mathbf{V}\right)=C e^{-\frac{1}{N_{0}}\left\|\mathbf{y}_{m}-\left(\mathbf{H}+\tilde{\beta} \mathbf{V} s_{m}\right) \mathbf{x}_{m}\right\|^{2}} .
$$

Thus, for given $\left\{\mathbf{y}_{m}\right\}$, the joint ML problem to estimate $\left\{\mathbf{x}_{m}, s_{m}\right\}$ as well as $\{\mathbf{V}, \tilde{\beta}\}$ can be formulated as

$$
\max _{\mathbf{V}, \tilde{\beta}} \max _{\mathbf{x}_{m} \in \mathcal{X}^{L}, s_{m} \in\{-1,+1\}} \prod_{m=0}^{M-1} f\left(\mathbf{y}_{m} \mid \mathbf{x}_{m}, s_{m}, \tilde{\beta}, \mathbf{V}\right) \text {. }
$$

Since the receiver mainly wants to detect $s_{m}$, we can also consider the following problem:

$$
\max _{\mathbf{V}, \tilde{\beta}} \max _{s_{m} \in\{-1,+1\}} \prod_{m=0}^{M-1} f\left(\mathbf{y}_{m} \mid s_{m}, \tilde{\beta}, \mathbf{V}\right),
$$

where

$$
f\left(\mathbf{y}_{m} \mid s_{m}, \tilde{\beta}, \mathbf{V}\right)=\sum_{\mathbf{x}_{m} \in \mathcal{X}^{L}} f\left(\mathbf{y}_{m} \mid \mathbf{x}_{m}, s_{m}, \tilde{\beta}, \mathbf{V}\right) \operatorname{Pr}\left(\mathbf{x}_{m}\right)
$$

Here, $\operatorname{Pr}\left(\mathbf{x}_{m}\right)$ is the probability that $\mathbf{x}_{m}$ is transmitted from the legacy OFDM transmitter. For equally likely OFDM symbols, $\operatorname{Pr}\left(\mathbf{x}_{m}\right)$ might be a constant for all $\mathbf{x}_{m} \in \mathcal{X}^{L}$. The computational complexity to solve (13) or 14] is prohibitively high for large $M$ and $L$. Thus, we need to resort to low-complexity approaches.

The EM algorithm can be used to iteratively solve (14) with low-complexity. Suppose that the following ML estimation is to be solved:

$$
\max _{\tilde{\beta}, \mathbf{V}} \prod_{m=0}^{M-1} f\left(\mathbf{y}_{m} \mid \tilde{\beta}, \mathbf{V}\right)
$$

where

$$
f\left(\mathbf{y}_{m} \mid \tilde{\beta}, \mathbf{V}\right)=\sum_{s_{m} \in\{-1,+1\}} f\left(\mathbf{y}_{m} \mid s_{m}, \tilde{\beta}, \mathbf{V}\right) .
$$

Taking the $s_{m}$ 's as missing variables and denoting by $\tilde{\mathbf{V}}^{(i)}$ the $i$ th estimate of $\tilde{\mathbf{V}}=\tilde{\beta} \mathbf{V}$, the E-step is given by

$$
Q\left(\tilde{\mathbf{V}} \mid \tilde{\mathbf{V}}^{(i)}\right)=\sum_{m} \mathbb{E}\left[\ln f\left(\mathbf{y}_{m}, s_{m} \mid \tilde{\mathbf{V}}\right) \mid \mathbf{y}_{m}, \tilde{\mathbf{V}}^{(i)}\right] .
$$

Then, the M-step is given by

$$
\begin{gathered}
\left\{\tilde{\beta}^{(i+1)}, \mathbf{V}^{(i+1)}\right\}=\operatorname{argmax}_{\tilde{\beta}, \mathbf{V}} Q\left(\tilde{\mathbf{V}} \mid \tilde{\mathbf{V}}^{(i)}\right) \\
\text { subject to } \tilde{\beta} \in \mathbb{C}, \quad \mathbf{V} \in \mathcal{D}^{+}
\end{gathered}
$$

where $\mathcal{D}^{+}$represents the set of diagonal matrices whose elements are nonnegative as $\mathbf{V}=\operatorname{diag}\left(\left|G_{0}\right|^{2}, \ldots,\left|G_{L-1}\right|^{2}\right)$. Clearly, $\tilde{\mathbf{V}}^{(i+1)}=\widetilde{\beta}^{(i+1)} \mathbf{V}^{(i+1)}$. It is noteworthy that $\tilde{\mathbf{V}}$ has the following constraint:

$$
\begin{gathered}
\tilde{\mathbf{V}} \in \tilde{\mathcal{V}}=\left\{\operatorname{diag}\left(\tilde{v}_{0}, \ldots, \tilde{v}_{L-1}\right) \mid v_{l}=\tilde{\beta} a_{l},\right. \\
\left.\tilde{\beta} \in \mathbb{C}, a_{l} \geq 0, l=0, \ldots, L-1\right\} .
\end{gathered}
$$

Thus, for any $\tilde{\mathbf{V}}=\operatorname{diag}\left(\tilde{v}_{0}, \ldots, \tilde{v}_{L-1}\right) \in \tilde{\mathcal{V}}$, the phases of the diagonal elements are the same, i.e.,

$$
\angle \tilde{v}_{l-1}=\angle \tilde{v}_{l}, l=1, \ldots, L-1,
$$

which is due to the matched-filter based transmission in the CAMF scheme. This constraint on $\tilde{\mathbf{V}}$ helps find a good estimate of $\tilde{\mathbf{V}}$.

The EM algorithm can also be applied to perform joint estimation and detection for the CUIF scheme, where $\beta \mathbf{G}$ is to be estimated instead of $\tilde{\mathbf{V}}$. Since the phases of $\beta \mathbf{G}$ are all different, there is no constraint such as 20 to be exploited for a better estimate of $\tilde{\mathbf{G}}$ to overcome Difficulty-II. As a result, as will be shown in Section VI, CUIF cannot perform better than CAMF.

\section{Approximations for Low-Complexity Implementations}

In this subsection, we discuss approximations for a lowcomplexity implementation of the EM algorithm for CAMF.

Let us consider the E-step in (18). It can be shown that

$$
\begin{aligned}
\ln f\left(\mathbf{y}_{m}, s_{m} \mid \tilde{\mathbf{V}}\right) & =\ln \left(f\left(\mathbf{y}_{m} \mid s_{m}, \tilde{\mathbf{V}}\right) \operatorname{Pr}\left(s_{m} \mid \tilde{\mathbf{V}}\right)\right) \\
& =\ln f\left(\mathbf{y}_{m} \mid s_{m}, \tilde{\mathbf{V}}\right)+\ln \operatorname{Pr}\left(s_{m}\right) .
\end{aligned}
$$

Since $s_{m}$ is equally likely, $\ln \operatorname{Pr}\left(s_{m}\right)$ becomes a constant. Thus, it can be shown that

$$
\begin{aligned}
& \mathbb{E}\left[\ln f\left(\mathbf{y}_{m}, s_{m} \mid \tilde{\mathbf{V}}\right) \mid \mathbf{y}_{m}, \tilde{\mathbf{V}}^{(i)}\right] \\
& =\ln f\left(\mathbf{y}_{m} \mid s_{m}, \tilde{\mathbf{V}}\right) \operatorname{Pr}\left(s_{m} \mid \mathbf{y}_{m}, \tilde{\mathbf{V}}^{(i)}\right)+C,
\end{aligned}
$$


where $C$ represents a constant. Since

$$
\left\|\mathbf{y}_{m}-\left(\mathbf{H}+\tilde{\beta} \mathbf{V} s_{m}\right) \mathbf{x}_{m}\right\|^{2}=\sum_{l=0}^{L-1}\left|y_{l, m}-\left(H_{l}+\tilde{\beta} V_{l} s_{m}\right) x_{l, m}\right|^{2},
$$

we can show that

$$
\begin{aligned}
\ell_{m}\left(s_{m}\right) & =\ln f\left(\mathbf{y}_{m} \mid s_{m}, \tilde{\mathbf{V}}\right) \\
& =\ln \sum_{\mathbf{x}_{m} \in \mathcal{X}} f\left(\mathbf{y}_{m} \mid \mathbf{x}_{m}, s_{m}, \tilde{\mathbf{V}}\right)+C \\
& \approx \ln f\left(\mathbf{y}_{m} \mid \hat{\mathbf{x}}_{m}\left(s_{m}\right), s_{m}, \tilde{\mathbf{V}}\right)+C \\
& =-\frac{1}{N_{0}} \sum_{l=0}^{L-1}\left|y_{l, m}-\left(H_{l}+\tilde{V}_{l} s_{m}\right) \hat{x}_{l, m}\left(s_{m}\right)\right|^{2},
\end{aligned}
$$

where

$$
\hat{x}_{l, m}\left(s_{m}\right)=\underset{x_{l, m} \in \mathcal{X}}{\operatorname{argmin}}\left|y_{l, m}-\left(H_{l}+\tilde{V}_{l}^{(i)} s_{m}\right) x_{l, m}\right|^{2} .
$$

In 23, we can see that the estimation of $\mathbf{x}_{m}$ is implicitly carried out for the carrier estimation. In particular, we can see that since $\mathbf{H}$ is known at the receiver, with the estimate of $\mathbf{x}_{m}$, the carrier estimation can be performed to address Difficulty-I in joint estimation and detection using the EM algorithm.

For convenience, let $p^{(i)}\left(s_{m}\right)=\operatorname{Pr}\left(s_{m} \mid \mathbf{y}_{m}, \tilde{\mathbf{V}}^{(i)}\right)=$ $\frac{e^{\ell_{m}\left(s_{m}\right)}}{e^{l_{m}(-1)}+e^{\ell_{m}(1)}}$. Then, substituting 23) into 22] and (18), we can show that

$$
Q\left(\tilde{\mathbf{V}} \mid \tilde{\mathbf{V}}^{(i)}\right) \approx-\frac{1}{N_{0}} \sum_{l, m} \sum_{s_{m} \in\{-1,+1\}} \psi_{l}\left(s_{m}\right) p^{(i)}\left(s_{m}\right)+C,
$$

where

$$
\psi_{l}\left(s_{m}\right)=\left|y_{l, m}-\left(H_{l}+\tilde{V}_{l} s_{m}\right) \hat{x}_{l, m}\left(s_{m}\right)\right|^{2} .
$$

The complexity to find the approximation of $Q\left(\tilde{\mathbf{V}} \mid \tilde{\mathbf{V}}^{(i)}\right)$ in 25) is mainly dependent on the detection of $x_{l, m}$ in 24. For given $\tilde{V}_{l}$ and $s_{m}$, the complexity to find $\hat{x}_{l, m}\left(s_{m}\right)$ is proportional to the size of $\mathcal{X}$, i.e., $|\mathcal{X}|$. Consequently, the resulting complexity becomes $O(2 M L|\mathcal{X}|)$.

The M-step in (19) is to find $\tilde{\beta}$ and $\mathbf{V}$, which is not easy to solve due to the constraints on $\tilde{\beta}$ and $\mathbf{V}$. However, if $\tilde{\mathbf{V}}=\tilde{\beta} \mathbf{V}$ is to be found (rather than $\tilde{\beta}$ and $\mathbf{V}$ ) without the constraint in 20), (19) is reduced to a minimization of a quadratic function of $V_{l}$. In particular, from 25], it can be shown that

$$
Q\left(\tilde{\mathbf{V}} \mid \tilde{\mathbf{V}}^{(i)}\right) \approx \sum_{l=0}^{L-1} Q_{l}\left(\tilde{V}_{l} \mid \tilde{\mathbf{V}}^{(i)}\right)+C,
$$

where

$$
Q_{l}\left(\tilde{V}_{l} \mid \tilde{\mathbf{V}}^{(i)}\right)=-\frac{1}{N_{0}} \sum_{m} \sum_{s_{m} \in\{-1,+1\}} \psi_{l}\left(s_{m}\right) p^{(i)}\left(s_{m}\right) .
$$

Since $\psi_{l}\left(s_{m}\right)$ is a quadratic function of $\tilde{V}_{l}$ as shown in 26, $Q_{l}\left(\tilde{V}_{l} \mid \tilde{\mathbf{V}}^{(i)}\right)$ is also a quadratic function of $\tilde{V}_{l}$. Therefore, $V_{l}$ that maximizes $Q_{l}\left(\tilde{V}_{l} \mid, \tilde{\mathbf{V}}^{(i)}\right)$ can be readily obtained, which is denoted by $\tilde{\nu}_{l}^{(i+1)}$. To impose the constraint in $[20$, from $\left\{\tilde{\nu}_{l}^{(i+1)}\right\}, \mathbf{V}^{(i+1)}$ can be found as

$$
\tilde{\mathbf{V}}^{(i+1)}=e^{j \hat{\theta}} \operatorname{diag}\left(\left|\tilde{v}_{0}^{(i+1)}\right|, \ldots,\left|\tilde{v}_{L-1}^{(i+1)}\right|\right) \in \tilde{\mathcal{V}}
$$

where $\theta$ is the average angle that is obtained as

$$
\hat{\theta}=\angle\left(\frac{1}{L} \sum_{l} \tilde{v}_{l}^{(i+1)}\right) .
$$

In summary, the joint estimation and detection can be performed as follows.

Co) Input: $\left\{\mathbf{y}_{m}\right\}$ and $\mathbf{H}$. Set an initial value for $\tilde{\mathbf{V}}^{(i)}$ with $i=0$,

C1) Compute $p^{(i)}\left(s_{m}\right)=\operatorname{Pr}\left(s_{m} \mid \mathbf{y}_{m}, \tilde{\mathbf{V}}^{(i)}\right)$ for all $m$ using (23).

C2) Find $Q_{l}\left(\tilde{V}_{l} \mid \tilde{\mathbf{V}}^{(i)}\right)$ for all $l$ and update $\mathbf{V}$ as in 28$)$.

C3) If $\left\|\tilde{\mathbf{V}}^{(i+1)}-\tilde{\mathbf{V}}^{(i)}\right\|_{\mathrm{F}}^{2}<\epsilon$ or $i>N_{\text {iter }}$ stop. Otherwise, move to $\mathbf{C 1}$ ) with $i \Rightarrow i+1$.

Here, $\epsilon>0$ is a pre-determined threshold and $N_{\text {iter }}$ represents the maximum number of iterations.

Note that the complexity for the M-step is also linearly proportional to $L$ (as $Q_{l}\left(\tilde{V}_{l} \mid \tilde{\mathbf{V}}^{(i)}\right.$ ) is a quadratic function of $\tilde{V}_{l}$ as mentioned earlier). Thus, the complexity per iteration (for both E- and M-steps) remains $O(M L|\mathcal{X}|)$. Consequently, the overall complexity of the EM algorithm for joint estimation and detection becomes $O\left(N_{\text {iter }} M L|\mathcal{X}|\right)$. As will be shown in Section VI, $N_{\text {iter }}$ is small (e.g., 5 iterations are required in general). Clearly, we can claim that joint estimation and detection can be carried out with a complexity (linearly) proportional to $M L$.

\section{Performance Analysis}

In this section, we analyze the performance of the CAMF and CUIF schemes under certain ideal conditions. In particular, throughout this section, we assume that the receiver not only knows its CSI, H, but also BD's CSI, i.e., G. In practice, since $\mathbf{G}$ is to be estimated in joint estimation and detection, the assumption leads to optimistic results.

\section{A. With Known OFDM Symbols}

For tractable analysis, we consider the following assumption.

A0) The receiver knows the OFDM symbols, i.e.,

$$
\hat{x}_{l, m}\left(s_{m}\right)=x_{l, m} \text {. }
$$

That is, the OFDM symbol is correctly known at the receiver, which results in an optimistic performance for the detection of the backscattered signal. Denote by $\mathbb{P}_{\text {cuif }}$ the probability of bit error or bit error rate (BER) of CUIF. Then, we have

$$
\begin{aligned}
\mathbb{P}_{\text {cuif }} & =\operatorname{Pr}\left(\|\mathbf{y}-(\mathbf{H}+\beta \mathbf{G}) \mathbf{x}\|^{2} \geq\|\mathbf{y}-(\mathbf{H}-\beta \mathbf{G}) \mathbf{x}\|^{2}\right) \\
& =\mathbb{E}\left[\mathbb{P}_{\text {cuif }}(\mathbf{G})\right],
\end{aligned}
$$

where

$$
\mathbb{P}_{\text {cuif }}(\mathbf{G})=\mathcal{Q}\left(\sqrt{\frac{\|2 \beta \mathbf{G x}\|^{2}}{2 N_{0}}}\right) .
$$

To derive a closed-form expression for the BER, we consider the following assumptions. 
A1) The coefficients of the CIR from the legacy OFDM transmitter to the BD are independent and

$$
g_{p} \sim \mathcal{C N}\left(0, \sigma_{g, p}^{2}\right)
$$

i.e., multipath Rayleigh fading is considered for the channel from the legacy OFDM transmitter to the BD.

A2) The amplitude of $x_{l, m}$ is constant, i.e., $\left|x_{l, m}\right|$ is the same for all $l$ and $m$ and $\left|x_{l, m}\right|^{2}=E_{x}$. For example, 4quadrature amplitude modulation (QAM) can be considered.

Under the assumptions of $\mathbf{A 0}, \mathbf{A 1}$, and $\mathbf{A 2}$, the conditional BER of CUIF can be given by

$$
\begin{aligned}
\mathbb{P}_{\text {cuif }}(\mathbf{G}) & =\operatorname{Pr}\left(\|\mathbf{n}\|^{2} \geq\|2 \beta \mathbf{G} \mathbf{x}+\mathbf{n}\|^{2} \mid \mathbf{G}\right) \\
& =\mathcal{Q}\left(\sqrt{\frac{2|\beta|^{2} \sum_{l}\left|G_{l} x_{l}\right|^{2}}{N_{0}}}\right) .
\end{aligned}
$$

Since $\sum_{l}\left|G_{l} x_{l}\right|^{2}=\sum_{l}\left|G_{l}\right|^{2} E_{x}=L E_{x} \sum_{p=0}^{P_{g}-1}\left|g_{p}\right|^{2}$, 32) is rewritten as

$$
\mathbb{P}_{\text {cuif }}(\mathbf{G})=\mathcal{Q}\left(\sqrt{2|\beta|^{2} L \gamma_{x} \sum_{p}\left|g_{p}\right|^{2}}\right)
$$

Thus, the BER of CUIF can be found as

$$
\begin{aligned}
\overline{\mathbb{P}}_{\text {cuif }}(L)= & \mathbb{E}\left[\mathcal{Q}\left(\sqrt{2 L|\beta|^{2} \gamma_{x} \sum_{p}\left|g_{p}\right|^{2}}\right)\right] \\
\approx & \frac{1}{12} \prod_{p=0}^{P_{g}-1} \frac{1}{1+L|\beta|^{2} \gamma_{x} \sigma_{g, p}^{2}} \\
& +\frac{1}{4} \prod_{p=0}^{P_{g}-1} \frac{1}{1+\frac{4}{3} L|\beta|^{2} \gamma_{x} \sigma_{g, p}^{2}},
\end{aligned}
$$

where $\gamma_{x}=\frac{E_{x}}{N_{0}}$. The approximation in 34 is due to [26], where $\mathcal{Q}(x)$ can be approximated as $\mathcal{Q}(x) \approx \frac{1}{12} e^{-\frac{x^{2}}{2}}+$ $\frac{1}{4} e^{-\frac{2 x^{2}}{3}}$.

If $\sigma_{g, p}^{2}=\sigma_{g}^{2}$ for all $p$, from [19] [27], the BER of CUIF can also be expressed as

$$
\overline{\mathbb{P}}_{\text {cuif }}(L)=\left(\frac{1-\mu}{2}\right)^{P_{g}} \sum_{p=0}^{P_{g}-1}\left(\begin{array}{c}
P_{g}-1+p \\
p
\end{array}\right)\left(\frac{1+\mu}{2}\right)^{p}
$$

where $\mu=\sqrt{\frac{L|\beta|^{2} \gamma_{x} \sigma_{g}^{2}}{1+L|\beta|^{2} \gamma_{x} \sigma_{g}^{2}}}$. From 34 and 35, it is clear that the diversity gain is $P_{g}$ and the BER decreases with $L$ as the bit energy (of scattered signals) increases. In addition, the BER decreases with the reflection coefficient, $\alpha$, or $|\beta|^{2}$.

Similarly, we can have the conditional BER of CAMF as follows:

$$
\mathbb{P}_{\text {camf }}(\mathbf{G})=\mathcal{Q}\left(\sqrt{\frac{\|2 \tilde{\beta} \mathbf{V} \mathbf{x}\|^{2}}{2 N_{0}}}\right)
$$

while the BER can be obtained by taking the mean with respect to $\mathbf{G}$, which is denoted by $\overline{\mathbb{P}}_{\text {camf }}(L)=\mathbb{E}\left[\mathbb{P}_{\text {camf }}(\mathbf{G})\right]$.
Theorem 1. Under the assumptions of A0, A1, and A2, it can be shown that

$$
\mathbb{P}_{\text {camf }}(\mathbf{G}) \leq \mathbb{P}_{\text {cuif }}(\mathbf{G})
$$

We have the equality in (37) if the $\left|G_{l}\right|^{2}$ 's are the same for all $l$.

Proof: From (29) and (36), for the inequality in (37), it is sufficient to show that

$$
\|\tilde{\beta} \mathbf{V} \mathbf{x}\|^{2} \geq\|\beta \mathbf{G} \mathbf{x}\|^{2}=|\beta|^{2} E_{x} L \sum_{p=0}^{P_{g}-1}\left|g_{p}\right|^{2} .
$$

Since $\kappa=\frac{\sqrt{L}}{\|\mathbf{g}\|}$, it can be shown that

$$
\begin{aligned}
\|\tilde{\beta} \mathbf{V} \mathbf{x}\|^{2} & =|\beta|^{2}|\kappa|^{2} E_{x} \sum_{l}\left|G_{l}\right|^{4} \\
& =\frac{|\beta|^{2} L E_{x} \sum_{l}\left|G_{l}\right|^{4}}{\sum_{l}\left|G_{l}\right|^{2}}=\frac{|\beta|^{2} E_{x} \sum_{l}\left|G_{l}\right|^{4}}{\sum_{p}\left|g_{p}\right|^{2}} .
\end{aligned}
$$

Using the Cauchy-Schwarz inequality, we can have the following inequality for a sequence $\left\{z_{1}, \ldots, z_{n}\right\}$ :

$$
\left|\sum_{i=1}^{n} z_{i}\right|^{2} \leq n \sum_{i=1}^{n}\left|z_{i}\right|^{2}
$$

This inequality can be applied to 39 and leads to

$$
\begin{aligned}
\| \tilde{\beta} \mathbf{V} \mathbf{x}||^{2} & \geq|\beta|^{2} E_{x} \frac{\left.\left.\left|\sum_{l}\right| G_{l}\right|^{2}\right|^{2}}{L \sum_{p}\left|g_{p}\right|^{2}} \\
& =|\beta|^{2} E_{x} L \sum_{p}\left|g_{p}\right|^{2},
\end{aligned}
$$

which is identical to 38.

For the equality in (38), let us consider the inequality in (40), where $z_{i}=\left|G_{i}\right|^{2}$. If all $z_{i}$ 's are the same, the inequality in (40) becomes the equality, which implies that $\|\tilde{\mathbf{V}} \mathbf{x}\|^{2}=$ $\|\beta \mathbf{G x}\|^{2}$ and $\mathbb{P}_{\text {camf }}(\mathbf{G})=\mathbb{P}_{\text {cuif }}(\mathbf{G})$. This completes the proof.

Although the BER of CAMF is lower than that of CUIF as in (37), the gap may not be significant if the $\left|G_{l}\right|^{2}$ 's are not significantly varying. In particular, if $\left|G_{l}\right|^{2} \approx A$ for all $l$ (e.g., near flat fading), as in Theorem 1, the BER of CAMF might be close to that of CUIF under ideal conditions (i.e., known $\mathbf{x}_{m}$ and $(\mathbf{H}, \mathbf{G})$ ).

\section{B. With Unknown OFDM Symbols}

Since the assumption of A0 leads to an exceedingly optimistic performance, we may need to take into account errors in detecting OFDM symbols. In this subsection, we consider an approach to find the performance of $\mathrm{CUIH}^{2}$ with detection errors of OFDM symbols at the receiver.

Since the OFDM symbol detection and backscattered signal detection are interrelated, the analysis with unknown OFDM symbols becomes involved. To avoid this difficulty, we consider a two-step approach. In the first step, we find the detection error probability of OFDM symbols under the

\footnotetext{
${ }^{2}$ Due to 37 , it is expected that the performance of CAMF is slightly better than that of CUIF in the case of unknown OFDM symbols as well.
} 
assumption that the backscattered signal becomes Gaussian interference. In the second step, we assume that the received signals corresponding to erroneously detected OFDM symbols are not used for the detection of backscattered signals. This implies that the number of subcarriers, $L$, decreases and results in a low SNR in the BER expression for CUIF, i.e., 34.

In the first step, since the backscattered signals are assumed to be Gaussian interference, under the assumption of $\mathbf{A 1}$, the variance of the background noise and interference becomes

$$
K_{0}=N_{0}+|\beta|^{2} E_{x} \sum_{p=0}^{P_{g}-1} \sigma_{g, p}^{2} .
$$

Since $x_{l, m}$ is a 4-QAM symbol (according to the assumption of A2), the BER of the OFDM symbol detection becomes

$$
\overline{\mathbb{P}}_{\text {ber }}=\mathbb{E}\left[\mathcal{Q}\left(\sqrt{\frac{2\left|H_{l}\right|^{2}}{K_{0}}}\right)\right] .
$$

As in A1, we can also consider multipath Rayleigh fading for $\mathbf{H}$, i.e., the $h_{p}$ 's are independent and

$$
h_{p} \sim \mathcal{C N}\left(0, \sigma_{h, p}^{2}\right) .
$$

Then, since $\left|H_{l}\right|^{2}$ becomes an exponential random variable, according to [19], the (average) BER of each OFDM data symbol, $x_{l, m}$, is given by

$$
\overline{\mathbb{P}}_{\text {ber }}=\frac{1}{2}\left(1-\sqrt{\frac{\gamma_{h}}{1+\gamma_{h}}}\right),
$$

where $\gamma_{h}=\frac{\sum_{p=0}^{P_{h}-1} \sigma_{h, p}^{2}}{K_{0}}$. If each bit error in $x_{l, m}$ is assumed to be independent, we have the symbol error rate (SER) of $x_{l, m}$ as follows

$$
\overline{\mathbb{P}}_{\text {ser }}=1-\left(1-\overline{\mathbb{P}}_{\text {ber }}\right)^{2} \text {. }
$$

This is the probability that the received signal through a subcarrier is not taken into account due to erroneous detection of $x_{l, m}$ for the backscattered signal detection. Thus, the probability that the number of subcarriers that can have correct OFDM symbol detection is $k$ given by

$$
\operatorname{Pr}(\hat{L}=k)=\left(\begin{array}{l}
L \\
k
\end{array}\right)\left(1-\overline{\mathbb{P}}_{\text {ser }}\right)^{k} \mathbb{P}_{\text {ser }}^{L-k},
$$

and the BER of CUIF with unknown OFDM symbols can be given by

$$
\overline{\mathbb{P}}_{\text {cuif }}=\sum_{k=0}^{L} \operatorname{Pr}(\hat{L}=k) \overline{\mathbb{P}}_{\text {cuif }}(k) .
$$

Note that since $\beta \mathbf{G}$ is assumed to be known at the receiver, (47) can also be seen as a lower-bound on the actual BER for the detection of backscattered signals by joint estimation and detection where $\beta \mathbf{G}$ is to be estimated. In addition, (47) may also be used for CAMF as an ideal performance, because the difference between $\mathbb{P}_{\text {camf }}(\mathbf{G})$ and $\mathbb{P}_{\text {cuif }}(\mathbf{G})$ may not be too large as mentioned earlier.

\section{Simulation Results}

In this section, we present simulation results with Rayleigh multipath channels for $\left\{h_{p}\right\}$ and $\left\{g_{p}\right\}$, where each channel coefficient is an independent zero-mean CSCG random variable and $\sigma_{h, p}^{2}=\sigma_{g, p}^{2}=\frac{1}{P}, p=0, \ldots, P-1$. Here, $P_{h}=P_{g}=P$. The channel coefficient from the BD to the receiver is assumed to be $f=e^{j \theta}$ for all the subcarriers, where $\theta$ is a random phase. In addition, we consider 4-QAM with $\mathcal{X}=\{ \pm 1 \pm j\}$. Since $E_{x}=2$, the SNR (for OFDM data symbols, $x_{l, m}$ ) or $E_{\mathrm{b}} / N_{0}$ is given as $\frac{1}{N_{0}}$, where $E_{\mathrm{b}}$ is the bit energy (which is a half of $E_{x}$ for 4-QAM).

For the EM algorithm, we consider a fixed number of iterations. For the initial values of the EM algorithm, we assume $p^{(0)}\left(s_{m}=+1\right)=p^{(0)}\left(s_{m}=-1\right)=\frac{1}{2}$ for all $m$ and $\tilde{V}_{l}^{(0)}=\alpha e^{j \hat{\theta}^{(0)}}$ for all $l$, where $\hat{\theta}^{(0)}$ is an initial estimate of $\theta=\angle(f)$. Note that the convergence of the EM algorithm depends on the initial values [21]. Thus, some initial values should be carefully chosen, in particular, the initial values of $\tilde{V}_{l}$, i.e., $\tilde{V}_{l}^{(0)}$, for CAMF. In this section, we assume that

$$
\left|\hat{\theta}^{(0)}-\theta\right| \leq \frac{\pi}{4}
$$

which means that the receiver is able to determine the phase of the channel coefficient, $f$, up to $\pm \frac{\pi}{4}$ difference through a rough initial estimate (to this end, the first bit of BD might be a pilot bit that is known to the receiver).

Fig. 3 shows the BERs of CAMF and CUIF as functions of SNR with $L=32, P=4, M=100$, and $\alpha^{2}=0.2$. For the EM algorithm, $N_{\text {iter }}$ is set to 5. The theoretical BERs of CUIF without and with OFDM symbol errors in (35) and (47), respectively, are also shown. Since they are obtained under the assumption that $\mathbf{G}$ is perfectly known at the receiver, both might be seen as lower-bounds. The BERs of CAMF and CUIF obtained from the EM algorithm decrease with the SNR, while CAMF performs better than CUIF as expected. It is noteworthy that the BER curve of CUIF has an error floor at a high SNR (i.e., $\geq 5 \mathrm{~dB}$ ), which might be due to the estimation error of $\mathbf{G}$. Since the BER of CAMF from the EM algorithm becomes close to the theoretical BER of CUIF with OFDM symbol errors in (47) as the SNR increases, we can confirm that the estimation of $\tilde{\mathbf{V}}$ can be reliably carried out in CAMF thanks to the matched-filtering at BD.

As mentioned earlier, CAMF can provide a better performance than CUIF because the estimation of $\tilde{\mathbf{V}}$ in CAMF can be more reliable than that of $\beta \mathbf{G}$ in CUIF. To see this, in Fig. 4. we show the estimates of $\tilde{\mathbf{V}}$ and $\beta \mathbf{G}$ for CAMF and CUIF, respectively, from the EM algorithm with $N_{\text {iter }}=5$ when $L=32, P=4, M=100$, SNR $=4 \mathrm{~dB}$, and $\alpha^{2}=0.2$. Exploiting the constraint that the phases of $\tilde{V}_{l}$ 's are the same (thanks to the matched-filtering at BD), it is shown that the EM algorithm for CAMF can provide a more reliable estimate of $\tilde{\mathbf{V}}$ than that of $\beta \mathbf{G}$ for CUIF, which results in a performance difference in Fig. 3 .

In order to see the performance improvement by increasing the number of iterations, $N_{\text {iter }}$, in the EM algorithm, the BERs of CAMF and CUIF are obtained as functions of $N_{\text {iter }}$ and shown in Fig. 5 when $L=32, P=4, M=100$, and $\alpha^{2}=$ 


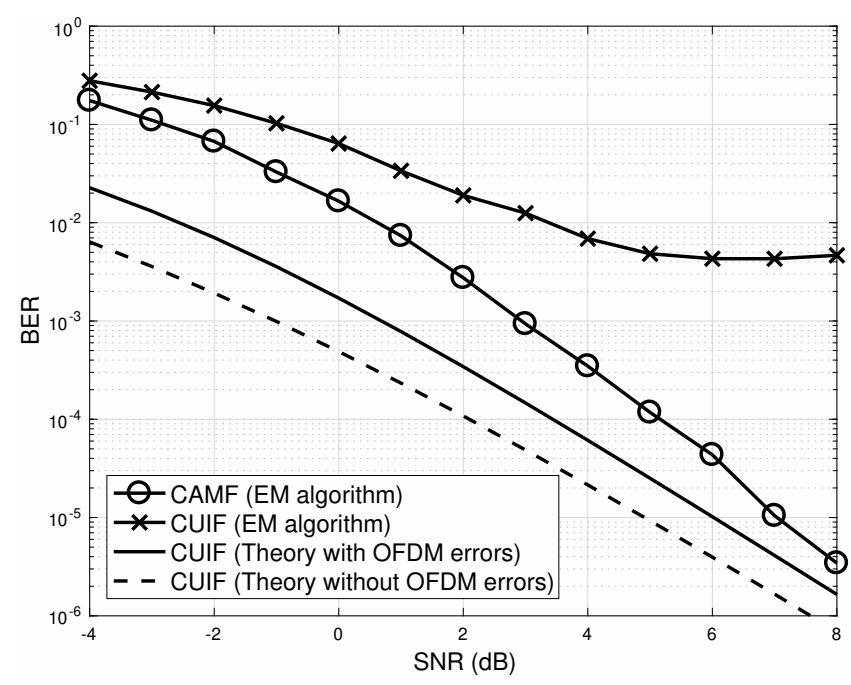

Fig. 3. BERs of CAMF and CUIF as functions of SNR with $L=32, P=4$, $M=100$, and $\alpha^{2}=0.2$. For the EM algorithm, we have $N_{\text {iter }}=5$.

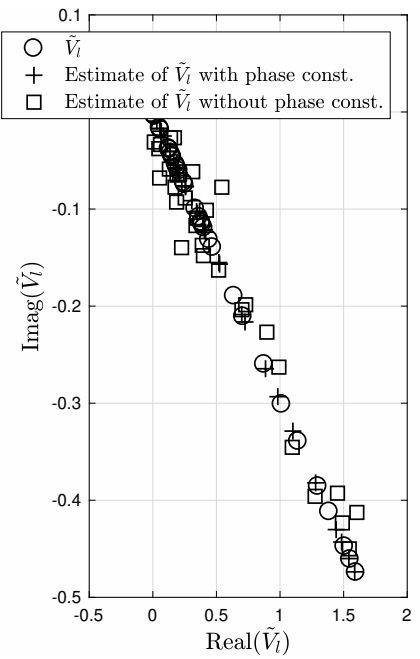

(a)

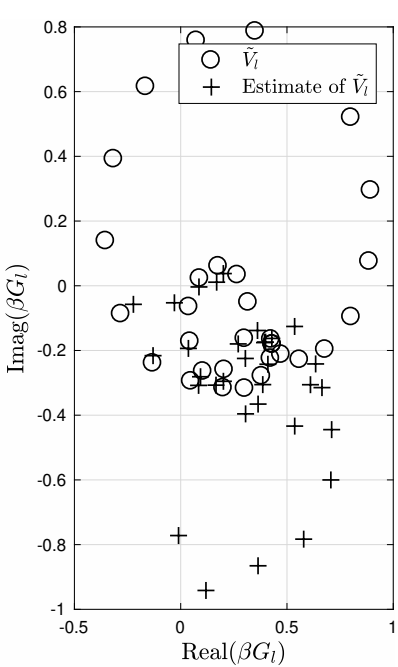

(b)
Fig. 4. The estimates of $\tilde{V}_{l}$ and $\beta G_{l}$ for CAMF and CUIF, respectively, from the EM algorithm with $N_{\text {iter }}=5$ when $L=32, P=4, M=100$, SNR $=4 \mathrm{~dB}$, and $\alpha^{2}=0.2$.

0.2. It is shown that $N_{\text {iter }}=5$ might be sufficient for CAMF (even at a high SNR, e.g., 6dB, as shown in Fig. 5(b)).

Fig. 6 shows the BERs of CAMF and CUIF as functions of $\alpha^{2}$ with $L=32, P=4, M=100$, and SNR $=6 \mathrm{~dB}$. For the EM algorithm, we have $N_{\text {iter }}=5$. In general, since the signal strength of scattered signals increases with $\alpha^{2}$, we expect a better performance for a larger $\alpha^{2}$ provided that the receiver is able to perform a reliable carrier estimation. This can happen with CAMF as the EM algorithm can perform reasonably well for joint estimation and detection. However, with CUIF, due to the difficulty in estimating $\beta \mathbf{G}$, joint estimation and detection cannot be properly carried out at the receiver and the performance becomes worse as $\alpha^{2}$ increases.

In Fig. 7, we show the BERs of CAMF and CUIF as functions of the length of OFDM symbol, $L$, with $P=4$, $\alpha^{2}=0.2, M=100$, and $\mathrm{SNR}=6 \mathrm{~dB}$. For the EM

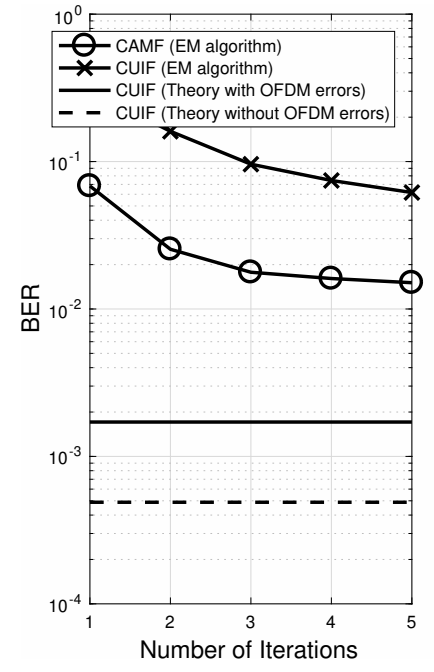

(a)

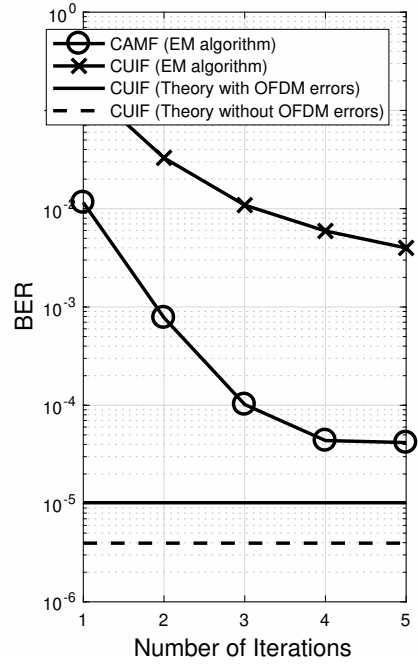

(b)
Fig. 5. BERs of CAMF and CUIF as functions of the number of iterations in the EM algorithm with $L=32, P=4, M=100$, and $\alpha^{2}=0.2$ : (a) $\mathrm{SNR}=0 \mathrm{~dB} ;$ (b) SNR $=6 \mathrm{~dB}$.

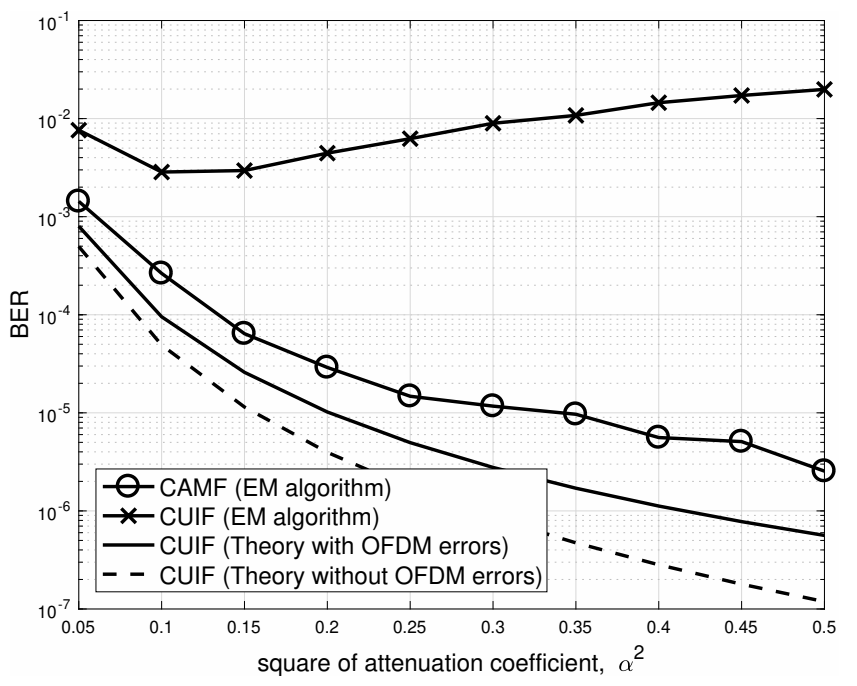

Fig. 6. BERs of CAMF and CUIF as functions of $\alpha^{2}$ with $L=32, P=4$, $M=100$, and SNR $=6 \mathrm{~dB}$. For the EM algorithm, we have $N_{\mathrm{iter}}=5$.

algorithm, $N_{\text {iter }}$ is set to 5 . Since $L$ is the bit energy for backscattered signals, a better performance is expected as $L$ increases. With CAMF, since joint estimation and detection can be successfully performed, it is shown that the BER decreases with $L$. However, with CUIF, due to the difficulty to estimate the phases of $\beta \mathbf{G}$, the performance improvement by increasing $L$ cannot be seen.

The impact of the number of multiple paths, $P$, on the BER is shown in Fig. 8 with $L=32, \alpha^{2}=0.2, M=100$, and $\mathrm{SNR}=6 \mathrm{~dB}$. For the EM algorithm, we have $N_{\text {iter }}=5$. Since the diversity gain increases with $P$ (which is shown in (34) and (35)), we expect a lower BER as $P$ increases, which is also confirmed by the simulation results in Fig. 8

As mentioned earlier, in general, it is difficult for the receiver to estimate BD's CSI, $\beta \mathbf{G}$, as it is not directly 


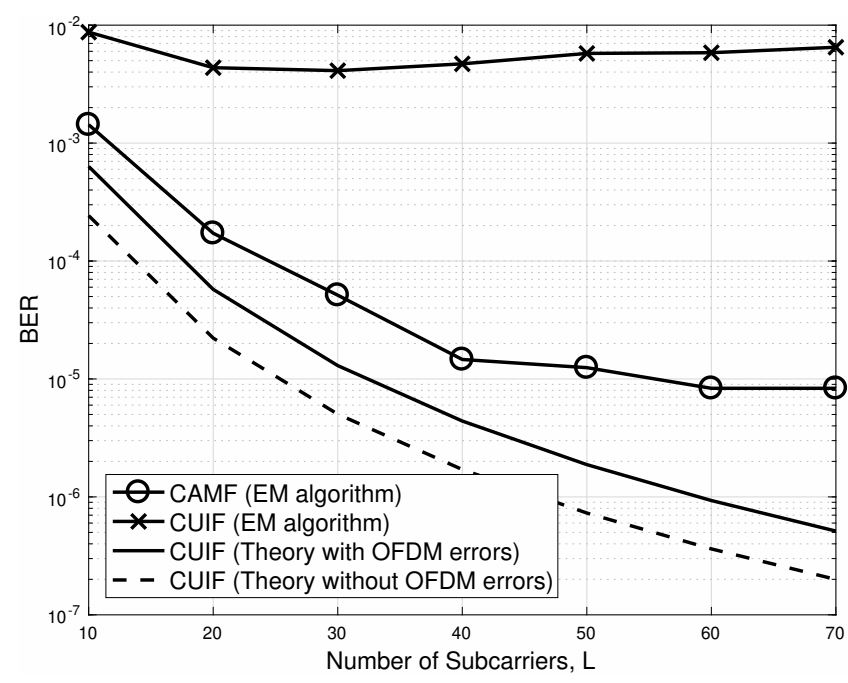

Fig. 7. BERs of CAMF and CUIF as functions of the length of OFDM symbol, $L$, with $P=4, \alpha^{2}=0.2, M=100$, and SNR $=6 \mathrm{~dB}$. For the EM algorithm, we have $N_{\text {iter }}=5$.

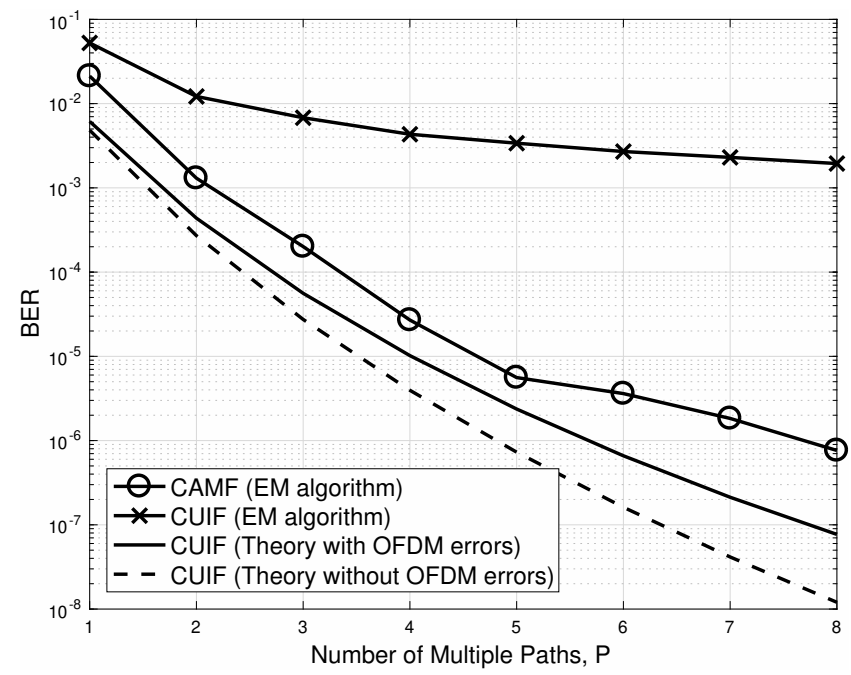

Fig. 8. BERs of CAMF and CUIF as functions of the length of CIR, $P$, with $L=32, \alpha^{2}=0.2, M=100$, and SNR $=6 \mathrm{~dB}$. For the EM algorithm, we have $N_{\text {iter }}=5$.

observable (due to OFDM symbols, $\mathbf{x}_{m}$, multiplied to $\mathbf{G}$ ). As a result, the performance of CUIF is not close to the theoretical one in 47) (with known BD's CSI) as shown in Figs. 3.8. On the other hand, in those simulation results, it is shown that the theoretical BER of CUIF in (47) can help predict the performance of the proposed CAMF scheme, although it serves as a lower-bound. This implies that the joint estimation and detection at the receiver is able to reliably estimate BD's CSI (i.e., $\tilde{\mathbf{V}}$ ) in CAMF thanks to the matched-filtering (as shown in Fig. 47. Therefore, we can expect a reliable detection of backscattered signals using CAMF at the expense of more signal processing operations at both the $\mathrm{BD}$ and receiver.

\section{CONCLUDING REMARKS}

In this paper, we proposed a novel modulation scheme, CAMF, for AmBC using OFDM carrier that can effectively exploit BD's CSI to help overcome the difficulty in performing joint estimation and detection at a receiver. Using the matchedfiltering (to BD's CSI), it became possible to enforce the forwarded OFDM signal by the BD to be coherently received at the receiver. As a result, a reliable estimate of BD's CSI has been available at the receiver and the backscattered signals have also been reliably detected. For low-complexity joint estimation and detection, the EM algorithm was considered with approximations. From simulation results, we have demonstrated that the performance of CAMF can be close to the theoretical one (which is obtained with known BD's CSI) thanks to the matched-filtering, while the performance became poor without the matched-filtering.

\section{REFERENCES}

[1] ITU-T, Y.2060: Overview of the Internet of things, June 2012.

[2] S. L. Keoh, S. S. Kumar, and H. Tschofenig, "Securing the Internet of Things: A standardization perspective," IEEE Internet of Things Journal, vol. 1, pp. 265-275, June 2014.

[3] S. Gollakota, M. S. Reynolds, J. R. Smith, and D. J. Wetherall, "The emergence of RF-powered computing," Computer, vol. 47, pp. 32-39, Jan 2014.

[4] D. M. Dobkin, The RF in RFID, Second Edition: UHF RFID in Practice. Newton, MA, USA: Newnes, 2nd ed., 2012.

[5] C. Boyer and S. Roy, "Backscatter communication and RFID: Coding, energy, and MIMO analysis," IEEE Trans. Communications, vol. 62, pp. 770-785, March 2014.

[6] J. Yu, W. Gong, J. Liu, L. Chen, and K. Wang, "On efficient tree-based tag search in large-scale rfid systems," IEEE/ACM Trans. Netw., vol. 27, pp. 42-55, Feb. 2019.

[7] Z. Luo, Q. Zhang, Y. Ma, M. Singh, and F. Adib, "3d backscatter localization for fine-grained robotics," in Proceedings of the 16th USENIX Conference on Networked Systems Design and Implementation, NSDI'19, (Berkeley, CA, USA), pp. 765-781, USENIX Association, 2019.

[8] Y. Ma, Z. Luo, C. Steiger, G. Traverso, and F. Adib, "Enabling deeptissue networking for miniature medical devices," in Proceedings of the 2018 Conference of the ACM Special Interest Group on Data Communication, SIGCOMM '18, (New York, NY, USA), pp. 417-431, ACM, 2018.

[9] V. Liu, A. Parks, V. Talla, S. Gollakota, D. Wetherall, and J. R. Smith, "Ambient backscatter: Wireless communication out of thin air," SIGCOMM Comput. Commun. Rev., vol. 43, pp. 39-50, Aug. 2013.

[10] B. Kellogg, A. Parks, S. Gollakota, J. R. Smith, and D. Wetherall, "Wi-fi backscatter: Internet connectivity for RF-powered devices," SIGCOMM Comput. Commun. Rev., vol. 44, pp. 607-618, Aug. 2014.

[11] G. Wang, F. Gao, R. Fan, and C. Tellambura, "Ambient backscatter communication systems: Detection and performance analysis," IEEE Trans. Communications, vol. 64, pp. 4836-4846, Nov 2016.

[12] D. Bharadia, K. R. Joshi, M. Kotaru, and S. Katti, "BackFi: High throughput WiFi backscatter," SIGCOMM Comput. Commun. Rev., vol. 45, pp. 283-296, Aug. 2015.

[13] C. Kang, W. Lee, Y. You, and H. Song, "Signal detection scheme in ambient backscatter system with multiple antennas," IEEE Access, vol. 5 , pp. 14543-14547, 2017.

[14] G. Yang, Q. Zhang, and Y. Liang, "Cooperative ambient backscatter communications for green internet-of-things," IEEE Internet of Things Journal, vol. 5, pp. 1116-1130, April 2018.

[15] G. Yang, D. Yuan, Y. Liang, R. Zhang, and V. C. M. Leung, "Optimal resource allocation in full-duplex ambient backscatter communication networks for wireless-powered IoT," IEEE Internet of Things Journal, pp. 1-1, 2019.

[16] G. Yang, Y. Liang, R. Zhang, and Y. Pei, "Modulation in the air: Backscatter communication over ambient OFDM carrier," IEEE Trans. Communications, vol. 66, pp. 1219-1233, March 2018.

[17] E. Dahlman, S. Parkvall, and J. Skold, 4G: LTE/LTE-Advanced for Mobile Broadband, 2nd Edition. Academic Press, 2013.

[18] I. Eizmendi, M. Velez, D. Gmez-Barquero, J. Morgade, V. BaenaLecuyer, M. Slimani, and J. Zoellner, "DVB-T2: The second generation of terrestrial digital video broadcasting system," IEEE Trans. Broadcasting, vol. 60, pp. 258-271, June 2014. 
[19] J. Proakis, Digital Communications. McGraw-Hill, fourth ed., 2000.

[20] A. P. Dempster, N. M. Laird, and D. B. Rubin, "Maximum likelihood from incomplete data via the em algorithm," J. of The Royal Statistical Society, Series B, vol. 39, no. 1, pp. 1-38, 1977.

[21] G. McLachlan and T. Krishnan, The EM Algorithm and Extensions. John Wiley \& Sons, 1997.

[22] J. Qian, F. Gao, G. Wang, S. Jin, and H. Zhu, "Semi-coherent detection and performance analysis for ambient backscatter system," IEEE Trans. Communications, vol. 65, pp. 5266-5279, Dec 2017.

[23] Y. S. Cho, J. Kim, W. Y. Yang, and C. G. Kang, MIMO-OFDM Wireless Communications with MATLAB. Wiley-IEEE Press, 2010.

[24] J. Choi, Adaptive and Iterative Signal Processing in Communications. Cambridge University Press, 2006.

[25] L. L. Scharf, Statistical Signal Processing: Detection, Estimation, and Time Series Analysis. Addison-Wesley, 1991.

[26] M. Chiani and D. Dardari, "Improved exponential bounds and approximation for the Q-function with application to average error probability computation," in Global Telecommunications Conference, 2002. GLOBECOM '02. IEEE, vol. 2, pp. 1399-1402 vol.2, Nov 2002.

[27] M. K. Simon and M. Alouini, Digital Communication over Fading Channels: A Unified Approach to Performance Analysis. John Willey, 2000. 